\title{
OPERATION AND CO-OPERATION IN THE GENERAL CATEGORIES
}

\author{
KISUKE TSUCHIDA
}

(Received August 2,1963)

1. Introduction. Let $\mathfrak{I}$ be a category consisting of the based topological spaces and based continuous maps. Eckmann and Hilton [2] defined an operation $\pi(B, F) \times \pi(B, \Omega Y) \rightarrow \pi(B, F)$ for a fibre map $P: X \rightarrow Y$ with fibre $F$ in $\mathfrak{I}$, where $\Omega Y$ denotes the loop space in $Y$ and $B$ any space. Dually they defined a co-operation $\rho^{*}: \pi(F, B) \times \pi(\Sigma Y, B) \rightarrow \pi(F, B)$ for a cofibre map $i: \mathrm{Y} \rightarrow X$ with cofibre $F$ in $\mathfrak{I}$, where $\Sigma Y$ denotes the suspension space of $Y$.

In this paper we try to define the corresponding one in the framework of the general categories.

Using a non-trivial semi-simplicial standard construction in the sense of Godement [4], Huber [5] developed a semi-simplicial homotpy theory in the framework of general categories.

Huber pointed out that the standard construction induces a fibration $K(Y)$ : $C Y \rightarrow Y$, (See $\S 3$ for the definition) and the fibre $\Omega Y$ of $K(Y)$ has the properties approximately corresponding to those of a loop space, and the dual standard construction induces a cofibration $K(X): X \rightarrow C X$ and the cofibre $\Sigma X$ of $K(X)$ has the formal properties of a suspension. Thus, we start from above consideration and try to study our problem.

In §3, by Huber's semi-simplicial homotopy, we shall define Kan homotopy groups in the general category, an operation $\rho_{*}: \pi_{n}\left(X, Y_{3}\right) \times \pi_{n}\left(X, \Omega Y_{1}\right) \rightarrow$ $\pi_{n}\left(X, Y_{3}\right)$ for a fibration sequence $Y_{3} \rightarrow Y_{2} \rightarrow Y_{1}$, and, in §5, dually a cooperation $\rho^{*}: \pi_{n}\left(X_{3}, Y\right) \times \pi_{n}\left(\Sigma X_{i}, Y\right) \rightarrow \pi_{n}\left(X_{3}, Y\right)$ for a cofibration sequence $X_{1} \rightarrow X_{2} \rightarrow X_{3}$.

Since Kan homotopy groups under the consideration are naturally isomorphic to Eckmann-Hilton homotopy groups which are higher by one dimension than the former [5], our operation and co-operation in the special case include the similar operation in [2] only in the group case. It is known that any map $v: X \rightarrow Y$ is factorized in the form $X \stackrel{u}{\rightarrow} E_{v} \stackrel{p}{\rightarrow} Y$, where $u$ is a homotopy equivalence and $p$ a fibre map with fibre $F_{v}$, and $F_{v}$ may also be interpreted as the fibre space over $X$ induced by the map $v$.

In $\$ 4$ we shall introduce a notion of the trace of morphisms corresponding the above $F_{v}$ and obtain the similar results in [2]. From theorems 4.3 and 4.5 in $\S 4$, there exists a fibration sequence $Y_{1} \rightarrow F_{v} \rightarrow Y_{2}$ for a morphism $v ; Y_{2} \rightarrow Y_{1}$ and so, in the category $\mathfrak{T}, F_{v}$ may be interpreted as the fibre space over $Y_{2}$ induced by the map $v: Y_{2} \rightarrow Y_{1}$. In the framework of general categries, theorems 4.8 and 4.11 play the corresponding roles in theorems 3.11 and 5.15 in [2] 
respectively. Last section is devoted to the dual discussions.

2. Preliminaries. A category $\AA$ consists of a non-empty class $\Omega$ of objects $X, Y, \cdots$ together with sets $\operatorname{Hom}(X, Y)$ of morphisms $f: X \rightarrow Y(X, Y \in \Re)$, and of an associative composition of morphisms $\circ: \operatorname{Hom}(X, Y) \times \operatorname{Hom}(Y, Z)$ $\rightarrow \operatorname{Hom}(X, Z),(f \cdot g) \rightarrow g \circ f$, which has both-sided identity $1_{X} \in(X, X)$.

An object $o \in \Omega$ is called a zero object if, for all $X$, the sets Hom $(X, 0)$ and $\operatorname{Hom}(o, X)$ consists of exactly one element 0 . If $\AA$ has a zero object, then in each set $\operatorname{Hom}(X, Y)$ we have a distinguished morphism 0 , called the zero morphism. A morphism $f: X \rightarrow Y$ is called an equivalence if there is a morphism $g: \mathrm{Y} \rightarrow X$ such that $g \circ f=1$ and $f \circ g=1$. A morphism $f$ is called an epimorphism if for any $Z$ and any $v_{i}: Y \rightarrow Z, i=1,2$, the relation $v_{1} \circ f=v_{2} \circ f$ implies $v_{1}=v_{2}$. A morphism $f$ is called a monomorphism if for any $W$ and any $w_{i}: W \rightarrow X, i=1,2$, the relation $f \circ w_{1}=f \circ w_{2}$ implies $w_{1}=w_{2}$.

Notice that if $f$ is an equivalence then $f$ is both epimorphism and monomorphism, but the converse is in general false.

Let $\mathscr{R}^{\prime}, \mathscr{\Omega}^{\prime \prime}, \mathfrak{M}^{\prime}$ and $\mathfrak{M}^{\prime \prime}$ be arbitrary categories. Let $F, G: \mathfrak{R}^{\prime} \rightarrow \mathscr{\Omega}^{\prime \prime}$ be covariant functors and $\theta: F \rightarrow G$ a functor morphism. Let $U: \mathscr{\Omega}^{\prime \prime} \rightarrow \mathfrak{M}^{\prime \prime}$ and $V: \mathfrak{M}^{\prime} \rightarrow \mathscr{\Omega}^{\prime}$ be covariant functors. According to (4), (5), we define $U * \theta * V:$ $U \circ F \circ V \rightarrow U \circ G \circ V$ by

$$
(U * \theta * V)(X)=U(\theta(V(X))) \quad \text { for } X \in \mathfrak{M}^{\prime} .
$$

If $U$ (or $V$ ) is the identity functor $I$, then we abbreviate $U * \theta * V$ to $\theta * V$ (or $U * \theta)$. Then the following formulas are valid for any covariant functors and any functor morphisms (cf. [4], [5]);

$$
\begin{aligned}
& (U \circ V) * \theta=U *(V * \theta) \\
& \theta *(U \circ V)=(\theta * U) * V \\
& (U * \theta) * V=U * \theta * V=U *(\theta * V) \\
& U *\left(\theta^{\prime} \circ \theta^{\prime \prime}\right) * V=\left(U * \theta^{\prime} * V\right) \circ\left(U * \theta^{\prime \prime} * V\right) \\
& (\psi * G) \circ(U * \varphi)=(V * \varphi) \circ(\psi * G)
\end{aligned}
$$

for any two functor morphisms $\varphi: F \rightarrow G, \psi: U \rightarrow V$.

Rule (5) may be remembered with the following commutative diagram:

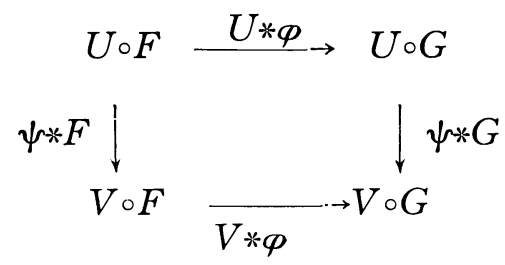

Throughout this paper we consider the categories with zero objects and zero morphisms and we assume that the functors preserve zero objects and zero 
morphisms.

Let $\AA$ be arbitrary category. Let $\{C, k, p\}$ be a standard construction in $\Omega$, i. e. $C: \Re \rightarrow \Re$ is a covariant functor, $k: C \rightarrow I$ and $p: C \rightarrow C \circ C$ are functor morphisms and the following two axioms are satisfied. (cf [4], [5])

$$
\begin{aligned}
& (k * C) \circ p=(C * k) \circ p=\text { identity, } \\
& (p * C) \circ p=(C * p) \circ p .
\end{aligned}
$$

Each standard construction $\{C, k, p\}$ generates a semi-simplical functor

$$
F_{*}=\left(F_{n}, d_{n}^{i}, s_{n}^{i}\right), \quad n \geqq 0
$$

i.e. a sequence of functors $F_{n}: \Re \rightarrow \Re$ together with faces and degeneracy morphisms

$$
\begin{aligned}
& d_{n}^{i}: F_{n} \rightarrow F_{n-1}, \\
& s_{n}^{i}: F_{n} \rightarrow F_{n+1},
\end{aligned}
$$

$F_{*}$ is dfined as follows: Let $C^{0}=I$ and $C^{n+1}=C_{\circ} C^{n}$. Then we put

$$
F_{n}=C^{n+1} \quad d_{n}^{i}=C^{i * k * C^{n-i}}, \quad s_{n}^{i}=C^{i} * p^{*} C^{n-i} .
$$

The faces and degeneracy morphisms satisfy the usual semi-simplicial commutation rules;

$$
\begin{array}{ll}
d^{i} d^{j}=d^{j-1} d^{i} & i<j \\
s^{i} s^{j}=s^{j+1} s^{i} & i \leqq j \\
d^{i} s^{j}=s^{j-1} d^{i} & i<j \\
d^{i} s^{i}=d^{i+1} s^{i}=\text { identiy } & \\
d^{i} s^{j}=s^{j} d^{i-1} & i>j+1 .
\end{array}
$$

If we apply the semi-simplicial functor $F_{*}$ to an object $Y \in \Re$, we obtain a semi-simplicial object $F_{*}(Y)=\left(F_{n}(Y), d^{i}(Y), s^{i}(Y)\right)$.

Let $\subseteq$ be the category of sets. If we now take the functor $\operatorname{Hom}(X$,$) :$ $\Re \rightarrow \subseteq$ with a fixed first argument $X$, we obtain a semi-simplicial complex

$$
K_{*}(X, Y)=\operatorname{Hom}\left(X, F_{*}(Y)\right)=\left(\operatorname{Hom}\left(X, F_{n}(Y)\right) d^{i}, s^{i}\right) \text {. }
$$

Then we have the Kan homotopy groups (cf [6]) of $K_{*}(X, Y)$ and we define $\pi_{n}(X, Y)=\pi_{n}\left(K_{*}(X, Y)\right) \quad(n \geqq 0)$. Here it is assumed that all the $K_{*}(X, Y)$ satisfy the Kan condition.

Now we define the kernel and the cokernel of a morphism. Let $u: X \rightarrow Y$ be a morphism.

A pair $(U, j)$ consisting of an object $U$ and of a monomorphism $j: U \rightarrow X$ is called a kernel of $u$, if$$
u \circ j=0
$$
(2) for each object $Z$ and each morphism: $v: Z \rightarrow X$ with $u \circ v=0$, there exists 
one and only one morphism $w: Z \rightarrow U$, so that $j \circ w=v$.

The definition of a cokernel is dually defined.

Throughout this paper, we shall be concerned with a category in which the kernel and the cokernel of morphisms always exist.

Let $K_{*}(X, Y)$ be the semi-simplicial complex induced by a standard construction $\{C, k, p\}$ in $\Re$.

Definition 2.1 ([5]). A morphism $\mathrm{u}: Y_{2} \rightarrow Y_{1}$ is called a fibration if the induced semi-simplicial map

$$
u_{*}: K_{*}\left(X, Y_{2}\right) \rightarrow K_{*}\left(X, Y_{1}\right)
$$

is a semi-simplicial fibration (cf [6]) for all $X \in \Re$.

If $C$ commutes with kernels (i.e. $C(\operatorname{Ker} u)=\operatorname{Ker} C(u)$ ), then the kernel $Y_{3}$ of $u$ will be called a fibre of $u$. Then the semi-simplicial fibre of $u_{*}$ may be identified with $K_{*}\left(X, Y_{3}\right)$.

PROposition 2.2 (Huber [5]). Let $\Omega$ be a category and let $\{C, k, \rho\}$ be a standard construction in $\Re$, such that the complexes $K_{*}(X, Y)$ are Kan complexes. Then

$$
\begin{array}{rl}
\text { the morphism } & k(Y): C Y \rightarrow Y \text { is a fibration, } \\
\pi_{n}(X, C Y)=0 & n \geqq 0 .
\end{array}
$$

If we denote the fibre of $k(Y)$ by $\Omega Y$, then we get the followin.

Proposition 2.3 (Huber [5]).

$$
\partial_{n+1}(k(Y)): \pi_{n+1}(X, Y) \rightarrow \pi_{n}(X, \Omega Y) \text { is isomorphic onto for } n>0 \text {. }
$$

3. Operations in Kan homotopy groups. Let $\Omega$ be a category and let $K_{*}(X, Y)$ be the semi-simplicial complex induced by a standard construction $\{C, k, p\}$ in $\Omega$. We suppose that all the $K_{*}(X, Y)$ satisfy Kan condition. Let $u: Y_{2} \rightarrow Y_{1}$ be a fibration and $\left(Y_{3}, v\right)$ its fibre. By proposition 2.2, $k\left(Y_{1}\right): C Y_{1} \rightarrow Y_{1}$ is a fibration. We take $(a, b) \in \pi_{n}\left(X, Y_{3}\right) \times \pi_{n}\left(X, \Omega Y_{1}\right)$. Let $\varphi: X \rightarrow C^{n+1} Y_{3}$ be a representative of the homotopy class $a \in \pi_{n}\left(X, Y_{3}\right)$. By proposition 2.3 , there exists only one $c \in \pi_{n+1}\left(X, Y_{1}\right)$ such that $\partial_{n+1}\left(k\left(Y_{1}\right)\right) c=b$. Let $\tau: X \rightarrow C^{n+2} Y_{1}$ be a representative of $c$.

Since $u_{*}: K_{*}\left(X, Y_{2}\right) \rightarrow K_{*}\left(X, Y_{1}\right)$ is a semi-simplicial fibration, there exists a $(n+1)$-simplex $\xi \in K_{*}\left(X, Y_{2}\right)$ such that $C^{n+2}(u) \xi=\tau$ and $d^{i} \xi=0$ for $i \neq n$ +1 . From the definition of the boundary homomorphism $\partial_{n+1}(u): \pi_{n+1}\left(X, Y_{1}\right)$ $\rightarrow \pi_{n}\left(Y, Y_{3}\right)$ for the fibration $u: Y_{2} \rightarrow Y_{1}, \partial_{n+1}(u) c$ is represented by a morphism $\chi: X \rightarrow C^{n+1} Y_{3}$ such that $C^{n+1}(v) \chi=d^{n+1} \xi$.

Now, if we take $n+1 n$-simplexes $\sigma_{i}=0(0 \leqq i \leqq n-2), \sigma_{n-1}=\phi$ and $\sigma_{n+1}=\chi$ in $K_{*}\left(X, Y_{3}\right)$, then we have $d^{j-1} \sigma_{i}=d^{i} \sigma_{j}$ for $i<j$ and $i, j \neq n$. Since 
$K_{*}\left(X, Y_{3}\right)$ is a Kan complex, then there exists a $(n+1)$-simplex $\eta \in K_{*}\left(X, Y_{3}\right)$ such that $d^{n-1} \eta=\varphi, d^{n+1} \eta=\chi$ and $d^{i} \eta=0(0 \leqq i \leqq n-2)$. From the definition of the product in Kan homotopy group $\pi_{n}\left(X, Y_{3}\right)$ (cf [6]), the homotopy class of $n$-simplex $d^{n} \eta \in K_{*}\left(X, Y_{3}\right)$ satisfy the relation $\left\{d^{n} \eta\right\}=\{\varphi\}\{\chi\}$ where \{\} denotes the homotopy class.

We now define an operation $\rho_{*}: \pi_{n}\left(X, Y_{3}\right) \times \pi_{n}\left(X, \Omega Y_{1}\right) \rightarrow \pi_{n}\left(X, Y_{3}\right)$ as

$$
\rho_{*}(a, b)=\left\{d^{n} \eta\right\}, \quad a \in \pi_{n}\left(X, Y_{3}\right), b \in \pi_{n}\left(X, \Omega Y_{1}\right) \text {. }
$$

Also the definition of $\rho_{*}$ may be written in the following abbreviated form:

$$
\rho_{*}(a, b)=a \cdot \partial_{n+1}(u) c,
$$

where $\partial_{n+1}\left(k\left(Y_{1}\right) c=b\right.$ and $\cdot$ denotes the product in Kan homotopy group $\pi_{n}\left(X, Y_{3}\right)$.

THEOREM 3. 1.

$$
\begin{array}{lr}
\rho_{*}(a, 0)=a & \left(a \in \pi_{n}\left(X, Y_{3}\right)\right) \\
\rho_{*}\left(a, b_{1} \cdot b_{2}\right)=\rho_{*}\left(\rho_{*}\left(a, b_{1}\right), b_{2}\right) & \left(b_{i} \in \pi_{n}\left(X, \Omega Y_{1}\right) i=1,2\right) .
\end{array}
$$

PROOF. Since (1) is evident we only prove (2). We have

$$
\begin{aligned}
& \rho_{*}\left(a, b_{1} \cdot b_{2}\right)=a \cdot \partial\left(c_{1} \cdot c_{2}\right)=a \cdot\left(\partial c_{1} \cdot \partial c_{2}\right) \\
& \rho_{*}\left(\rho_{*}\left(a, b_{1}\right), b_{2}\right)=\rho_{*}\left(a \cdot \partial c_{1}, b_{2}\right)=\left(a \cdot \partial c_{1}\right) \cdot \partial c_{2} .
\end{aligned}
$$

Hence (2) follows from the associativity in Kan homotopy group.

4. The trace of morphism. In this section we shall consider a category $\Re$ with direct products. Here we recall the defintion of a direct product in [3].

A direct product of the objects $A_{1}, A_{2}$ is an object $A_{1} \times A_{2}$ and a system of morphisms $p_{i}: A_{1} \times A_{2} \rightarrow A_{i}, i=1,2$, with the property:

(D). For any object $X$ of $\Omega$ and any system of morphisms $f_{i}: X \rightarrow A_{i}, i=$ 1,2 , there exists a unique morphism $\mathrm{f}: X \rightarrow A_{1} \times A_{2}$ with $p_{i} \cdot f=f_{i}$.

The morphisms $p_{i}$ are called the projection of $A_{1} \times A_{2}$, and morphisms $f_{i}$ are called the component of $f$; we write $f=\left\{f_{1}, f_{2}\right\}$, so that

$$
p_{i}\left\{f_{1}, f_{2}\right\}=f_{i} \text {. }
$$

Let $\left(B \times B, p_{1}^{\prime}, p_{2}^{\prime}\right)$ be a direct product of the object $B$ 's and let morphisms $f_{i}: A_{i} \rightarrow B$ be given, $i=1,2$. The morphism

$$
\left\{f_{1} p_{1}, f_{2} p_{2}\right\}: A_{1} \times A_{2} \rightarrow B \times B
$$

will be written $f_{1} \times f_{2}$. Then also we have

$$
p_{1}^{\prime}\left(f_{1} \times f_{2}\right)=f_{1} p_{1} \text { and } p_{2}^{\prime}\left(f_{1} \times f_{2}\right)=f_{2} p_{2} \text {. }
$$

Definition 4. 1 . The trace of $f_{1} \times f_{2}: A_{1} \times A_{2} \rightarrow B \times B$ is a pair $(Q, \iota)$ consisting of an object $Q$ and monomorphism $\iota: Q \rightarrow A_{1} \times A_{2}$ satisfying the conditions :

$$
\left.p_{1}^{\prime}\left(f_{1} \times f_{2}\right) \iota=p_{2}^{\prime}\left(f_{1} \times f_{2}\right) \iota \quad \text { (or equivalenty } f_{1} p_{1} \iota=f_{2} p_{2} \iota\right)
$$


(ii) if $D$ is any object of $\mathscr{\Re}$ and $h: D \rightarrow A_{1} \times A_{2}$ is a morphism with the property $p_{1}^{\prime}\left(f_{1} \times f_{2}\right) h=p_{2}^{\prime}\left(f_{1} \times f_{2}\right) h$ (or equivalently $\left.f_{1} p_{1} h=f_{2} p_{2} h\right)$,

then $h$ admits a unique factorization $D \rightarrow Q \stackrel{\iota}{\rightarrow} A_{1} \times A_{2}$.

For example, if $\mathscr{R}$ is a category of sets with base points, then the trace of $f_{1} \times f_{2}$ is $Q=\left\{\left(a_{1}, a_{2}\right) \in A_{1} \times A_{2} ; f_{1} a_{1}=f_{2} a_{2}\right\}$.

Now we return to the notation in $\$ 3$. Let $\{C, k, p\}$ be a standard construction in $\Re$ and we assume that functor $C$ is $D$-functor in the sense of $[3 ; \S 3]$, i. e.

$C\left(A_{1} \times A_{2}\right)=C\left(A_{1}\right) \times C\left(A_{2}\right)$ and $C\left(p_{i}\right): C\left(A_{1} \times A_{2}\right) \rightarrow C\left(A_{i}\right), i=1,2$, are projections.

Moreover we assume that if $f: A \rightarrow B$ is a monomorphism, then $C(f)$ : $C(A) \rightarrow C(B)$ is so.

Let $k(Y): C Y_{1} \rightarrow Y_{1}$ be a morphism in $\S 2$ and $v: Y_{2} \rightarrow Y_{1}$ any morphism. Then we denote the trace of $k\left(Y_{1}\right) \times v$ by $\left(F_{v}, j\right)$. Let $q_{1}: C Y_{1} \times Y_{2} \rightarrow C Y_{1}$ and $q_{2}: C Y_{1} \times Y_{2} \rightarrow Y_{2}$ be projections. Then we have the following commutative diagram :

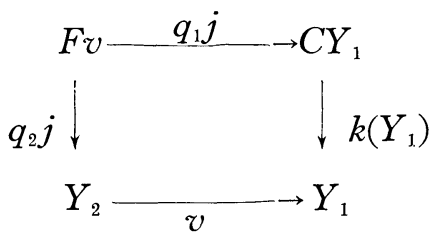

TheOREM 4. 3. For any morphism $v: Y_{2} \rightarrow Y_{1}, q_{2} j: F v \rightarrow Y_{2}$ is a fibration.

PROOF. Let $n(n-1)$-simplexes $\sigma_{0}, \cdots, \sigma_{k-1}, \sigma_{k+1}, \cdots, \sigma_{n} \in K_{*}\left(X, F_{v}\right)$ satisfy $d^{j-1} \sigma_{i}=d^{i} \sigma_{j}$ for $i<j$ and $i, j \neq k$ and let an $n$-simplex $\tau \in K_{*}(X, Y)$ satisfy $d^{i} \tau=C^{n}\left(q_{2} j\right) \sigma_{i}$ for $i \neq k$. If we consider $C^{n}\left(q_{1} j\right) \sigma_{i}{ }^{\prime} \mathrm{s}(i \neq k)$ and $C^{n+1}(v) \tau$, then we have

and

$$
d^{n}\left(C Y_{1}\right) C^{n}\left(q_{1} j\right) \sigma_{j}=d^{j-1}\left(C Y_{1}\right) C^{n}\left(q_{1} j\right) \sigma_{i} \quad \text { for } i<j \text { and } i, j \neq k
$$

$$
C^{n}\left(k\left(Y_{1}\right) C^{n}\left(q_{1} j\right)=d^{i}\left(Y_{1}\right) C^{n+1}(v) \tau .\right.
$$

By Proposition 2.2, $k\left(Y_{1}\right): C Y_{1} \rightarrow Y_{1}$ is a fibration and hence there exists a $n$-simplex $\sigma \in K_{*}\left(X, C Y_{1}\right)$ such that $d^{i}\left(C Y_{1}\right) \sigma=C^{n}\left(q_{1} j\right) \sigma_{i}$ for $i \neq k$ and $C^{n+1}\left(k\left(Y_{1}\right)\right) \sigma=C^{n+1}(v) \tau$. Since $C^{n+1}\left(C Y_{1} \times Y_{2}\right)=C^{n+1}\left(C Y_{1}\right) \times C^{n+1}\left(Y_{2}\right)$, there exists a morphism $\xi: X \rightarrow C^{n+1}\left(C Y_{1} \times Y_{2}\right)$ such that $C^{n+1}\left(q_{1}\right) \xi=\sigma$ and $C^{n+1}\left(q_{2}\right) \xi$ $=\tau$. But $C^{n+1}\left(k\left(Y_{1}\right)\right) C^{n+1}\left(q_{1}\right) \xi=C^{n+1}(v) C^{n+1}\left(q_{2}\right) \xi$. Hence, from the property

(ii) in Definition $5.1, \xi$ may be factorized in the form:

$$
X \stackrel{\eta}{\longrightarrow} C^{n+1}(F v) \stackrel{C^{n+1}(j)}{\longrightarrow} C^{n+1}\left(C Y_{1} \times Y_{2}\right) \text {. }
$$

Now, consider an $n$-simplex $\eta \in(X, F v)$, we have

$$
C^{n}\left(q_{1}\right) C^{n}(j) d^{i} \eta=C^{n}\left(q_{1}\right) C^{n}(j) \sigma_{i}
$$




$$
C^{n}\left(q_{2}\right) C^{n}(j) d^{i} \eta=C^{n}\left(q_{2}\right) C^{n}(j) \sigma_{i} \quad \text { for } i \neq k .
$$

Since $C^{n}\left(q_{1}\right)\left(C^{n}\left(q_{2}\right)\right)$ is the projection $C^{n}\left(Y_{1} \times Y_{2}\right) \rightarrow C^{n}\left(C Y_{1}\right)\left(C^{n}\left(Y_{2}\right)\right)$, by the property of the direct product, we have

$$
C^{n}(j) d^{i} \eta=C^{n}(j) \sigma_{i} \quad i \neq k .
$$

As $j$ is a monomorphism, $C^{n}(j)$ is so and hence $d^{i} \eta=\sigma_{i}$ for $i \neq k$. Also evidently we have $C^{n+1}\left(q_{2} j\right) \eta=\tau$.

Q. E. D.

THEOREM 4.4. If $v: Y_{2} \rightarrow Y_{1}$ is a fibration, $q_{1} j: F v \rightarrow C Y_{1}$ is so.

The proof of Theorem 4.4 is the same as that of Theorem 4.3 and we omit the proof.

In the following we suppose that the kernel of every fibration commutes with $C$, i. e. every fibration has a fibre in the sense of the definition of $\$ 2$.

THEOREM 4.5. The fibre of the fibration $q_{2} j: F_{v} \rightarrow Y_{2}$ is equivalent with $\Omega Y_{1}$, where $\Omega Y_{1}$ is the fibre of the fibration $k\left(Y_{1}\right): C Y_{1} \rightarrow Y_{1}$.

ProOF. We denote the fibre of the fibration $q_{2} j$ by $(K, k)$. Consider the following diagram :

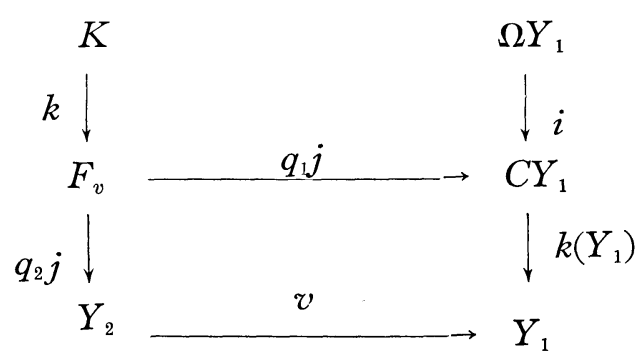

Since $k\left(Y_{1}\right) q_{1} j k=v q_{2} j k=0$, there exists a morphism $m: K \rightarrow \Omega Y_{1}$ such that $i m=q_{1} j k$. Let $\iota_{1}: C Y_{1} \rightarrow C Y_{1} \times Y_{2}$ be a mophism satisfying $q_{1} \iota_{1}=$ identity and $q_{2} \iota_{1}=0$. (Such a morphism surely exists from the property of the direct product.)

Consider a morphism $\iota_{1} \circ i: \Omega Y_{1} \rightarrow C Y_{1} \times Y_{2}$, then we have

$$
p_{1}\left(k\left(Y_{1}\right) \times v\right) \iota_{1} i=p_{2}\left(k\left(Y_{1}\right) \times v\right) \iota_{1} i .
$$

Hence, by the property of trace $F_{v}, \iota_{1} i$ is factorized in the form:

$$
\Omega \stackrel{w}{Y}_{1} \longrightarrow \stackrel{j}{F}_{v} \longrightarrow C Y_{1} \times Y_{2}
$$

$q_{2} j w=q_{2} \iota_{1} i=0$ implies the existence of a morphism $n: \Omega Y_{1} \rightarrow K$ such that $k n=w$. Then $i m n=q_{1} j k n=q_{1} j w=q_{1} \iota_{1} i=i$ and $m n=$ identity, for $i$ is a monomophism. Also we have

$$
q_{1} j k n m=q_{1} \iota_{1} i m=q_{1} j k
$$


and

$$
q_{2} j k n m=0=q_{2} j k .
$$

Hence, by the property of the direct product, we have $j k n m=j k$, in which both $J$ and $k$ are monomorphisms. Hence $n m=$ identity. Thus $K$ is equivalent with $\Omega Y_{1}$.

Q.E. D.

Henceforth we shall identity $K$ with $\Omega Y_{1}$ and we have the following fibration sequence :

$$
\Omega Y_{1} \stackrel{k}{\longrightarrow} F_{v} \stackrel{q_{2} j}{\longrightarrow} Y_{2} .
$$

Next let $v: Y_{2} \rightarrow Y_{1}$ be a fibration with fibre $\left(Y_{3}, u\right)$. Then the following theorem may be proved analogously as in Theorem 4.2.

THEOREM 4.6. If a sequence $Y_{3} \rightarrow Y_{2} \rightarrow Y_{1}$ is a fibration sequence, then a sequence $Y_{3} \rightarrow F_{v} \rightarrow C Y_{1}$ is so.

In virtue of Theorems 4.5 and 4.6, if $v: Y_{2} \rightarrow Y_{1}$ is a fibration with fibre $\left(Y_{3}, u\right)$, then we have the following commutative diagram:

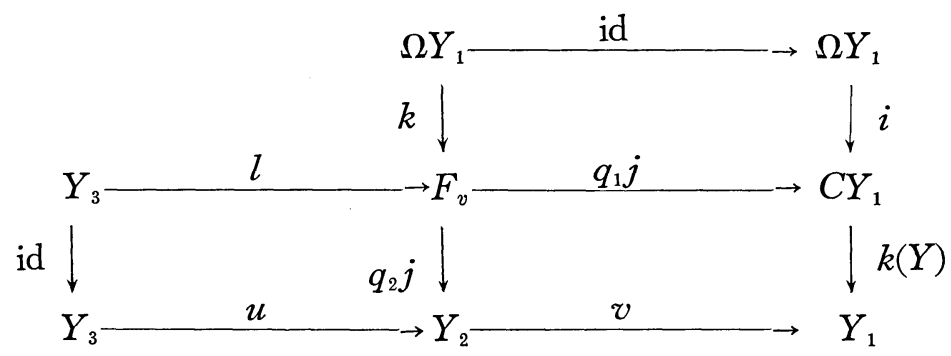

THEOREM 4.8. If $v: Y_{2} \rightarrow Y_{1}$ is a fibration with fibre $\left(Y_{3}, u\right)$. then

$$
l_{*}: \pi_{n}\left(X, Y_{3}\right) \rightarrow \pi_{n}\left(X, F_{v}\right)
$$

is an isomophism for $n \geqq 0$.

PROOF. The result follows from the Kan homotopy exact sequence of the fibration $q_{1} j: F_{v} \rightarrow C Y_{1}$ and proposition 2.2.

Note that Theorem 4.8 is also obtained from the next theorem 4.9 and the five lemma.

THEOREM 4.9 If $v: Y_{2} \rightarrow Y_{1}$ is a fibration with fibre $\left(Y_{3}, u\right)$, then the following diagram is commutative; 


$$
\begin{aligned}
& \longrightarrow \pi_{n+1}\left(X, Y_{1}\right) \stackrel{\partial(v)}{\longrightarrow} \pi_{n}\left(X, Y_{3}\right) \stackrel{u_{*}}{\longrightarrow} \pi_{n}\left(X, Y_{2}\right) \stackrel{v_{*}}{\longrightarrow} \pi_{n}\left(X, Y_{1}\right) \longrightarrow
\end{aligned}
$$

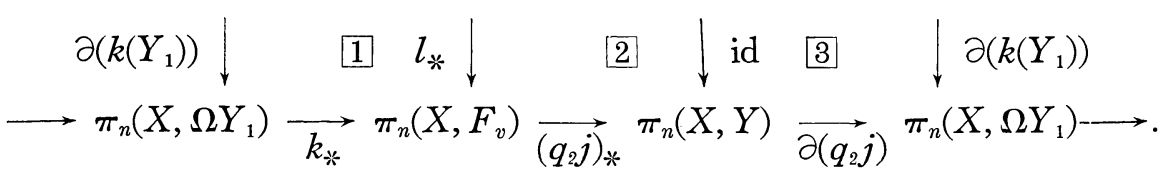

Here the upper sequence is Kan homotopy exact sequence of the fibration $v$ : $Y_{2} \rightarrow Y_{1}$ (cf [5] [6]) and the lower is that of the fibration $q_{2} j: F_{v} \rightarrow Y_{2}$.

LEMMA. Let $Y_{3} \stackrel{u}{\longrightarrow} Y_{2} \stackrel{v}{\longrightarrow} Y_{1}$ be a fibration sequence, then the boundary homomorphism $\partial(v): \pi_{n+1}\left(X, Y_{1}\right) \rightarrow \pi_{n}\left(X, Y_{3}\right)$ may be defined as follows: let $a \in \pi_{n+1}\left(X, Y_{1}\right)$ be represented by a morphism $\tau: X \rightarrow C^{n+2}\left(Y_{1}\right)$ such that $d^{i} \tau$ $=0(0 \leqq i \leqq n+1)$, then $\partial(v) a$ is represented by a morphism $\alpha: X \rightarrow C^{n+1}\left(Y_{3}\right)$ determined by the relation: $C^{n+2}(v) \xi=\tau, d \xi=0$ for $i \neq n$ and $d^{n} \xi=C^{n+1}(u) \alpha$, where $\xi: X \rightarrow C^{n+2}\left(Y_{2}\right)$ exists since $v: Y_{2} \rightarrow Y_{1}$ is a fibration,

PROOF. According to the usual definition of the boundary homomorphism, $\partial(v) a$ is represented by a morphisms $\beta: X \rightarrow C^{n+1}\left(Y_{3}\right)$ (cf [6]) determined by the relation; $C^{n+2}(v) \eta=\tau, d^{i} \eta=0$ for $i \neq n+1$ and $d^{n+1} \eta=C^{n+1}(u) \beta$, where $\eta: X \rightarrow C^{n+2}\left(Y_{2}\right)$ exists since $v: Y_{2} \rightarrow Y_{1}$ is a fibration.

In order to prove the lemma we have only to prove the existence of a morphism $\omega: X \rightarrow C^{n+2}\left(Y_{2}\right)$ satisfying $C^{n+2}(v) \omega=0, d^{n} \omega=d^{n+1} \eta, \quad d^{n+1} \omega=d^{n} \xi$ and $d^{i} \omega=0 i<n$. Take $n+2 n+1$-simplexes in $K_{*}(X, Y) \sigma_{i}=0,0 \leqq i \leqq n$ $-1, \sigma_{n}=s^{n+1} d^{n+1} \eta, \sigma_{n+1}=s^{n+1} d^{n} \xi$, then $d^{i} \sigma_{j}=d^{j-1} \sigma_{i}$ for $i<j, i, j \neq n+2$ and $C^{n+2}(v) \sigma_{i}=0$ for $i<n+2$. Since $v: Y_{2} \rightarrow Y_{1}$ is a fibration, then there exists $\zeta: X \rightarrow C^{n+3}\left(Y_{2}\right)$ such that $C^{n+3}(v) \zeta=0 \quad d^{n} \zeta=s^{n+1} d^{n+1} \eta \quad d^{n+1} \zeta=s^{n+1} d^{n} \xi$ and $d^{i} \zeta=0$ for $i<n$. If we consider $d^{n+1} \zeta: X \rightarrow C^{n+2}\left(Y_{2}\right)$, we have

$$
\begin{aligned}
& C^{n+2}(v) d^{n+2} \zeta=0, \\
& d^{n} d^{n+2} \zeta=d^{n+1} d^{n} \zeta=d^{n+1} s^{n+1} d^{n+1} \eta=d^{n+1} \eta, \\
& d^{n+1} d^{n+2} \zeta=d^{n+1} d^{n+1} \zeta=d^{n+1} s^{n+1} d^{n} \xi=d^{n} \xi, \\
& d^{i} d^{n+2} \zeta=d^{n+1} d^{i} \zeta=0(i<n) .
\end{aligned}
$$

Hence $d^{n+2} \zeta: X \rightarrow C^{n+2}\left(Y_{2}\right)$ is a required morphism.

The PROOF OF THEOREM 4.9.

i) Commutativity of 1 . Let $\tau: X \rightarrow C^{n+2}\left(Y_{1}\right)$ be a representative of $a \in$ $\pi_{n+1}\left(X, Y_{1}\right)$.

Since $k\left(Y_{1}\right): C Y_{1} \rightarrow Y_{1}$ is a fibration, there exists a morphism $\eta: X \rightarrow C^{n+2}$ $\left(C Y_{1}\right)$ such that $C^{n+2}\left(k\left(Y_{1}\right)\right) \eta=\tau$ and $d^{i} \eta=0$ for $i \leqq n$. Then we have a morphism $\psi: X \rightarrow C^{n+1}\left(\Omega Y_{1}\right)$ satisfying $C^{n+1}(i) \psi=d^{n+1} \eta$.

Since $q_{1} j: F_{v} \rightarrow C Y_{1}$ is a fibration and $C^{n+1}\left(q_{1} j\right) C^{n+1}(k) \psi=C^{n+1}(i) \psi=d^{n+1} \eta$, 
there exists a morphism $\alpha: X \rightarrow C^{n+2}\left(F_{v}\right)$ such that $C^{n+2}\left(q_{1} j\right) \alpha=\eta, \quad d^{i} \alpha=0$ for $i<n$, and $d^{n+1} \alpha=C^{n+1}(k) \psi$. Then we have $C^{n+2}(v) C^{n+2}\left(q_{2} j\right) \alpha=C^{n+2}\left(k\left(Y_{1}\right)\right)$ $C^{n+2}\left(q_{1} j\right) \alpha=C^{n+2}\left(k\left(Y_{1}\right)\right) \eta=\tau$ and $d^{i} C^{n+2}\left(q_{2} j\right) \alpha=0$ for $i \neq n$.

Since $C^{n+1}(v) C^{n+1}\left(q_{2} j\right)^{n} d \alpha=0$ and $v: Y_{2} \rightarrow Y_{1}$ is a fibration, by the lemma, the morphism $\zeta: X \rightarrow C^{n+1}\left(Y_{3}\right)$ determined by $C^{n+1}\left(q_{2} j\right) d^{n} \alpha=C^{n+1}(u) \zeta$ may be considered as a representative of $\partial(v) a$.

Since

$$
\begin{aligned}
& C^{n+1}\left(q_{2} j\right) C^{n+1}(l) \zeta=C^{n+1}(u) \zeta=C^{n+1}\left(q_{2} j\right) d^{n} \alpha, \\
& C^{n+1}\left(q_{1} j\right) C^{n+1}(l) \zeta=0 \quad \text { and } \\
& C^{n+1}\left(q_{1} j\right) d^{n} \alpha=d^{n} \eta=0,
\end{aligned}
$$

by the property of the direct product, we have $C^{n+1}(j) C^{n+1}(l) \zeta=C^{n+1}(j) d^{n} \alpha$. But $j$ is a monomophism and hence $d^{n} \alpha=C^{n+1}(l) \zeta$.

Accordingly $\alpha: X \rightarrow C^{n+2}\left(F_{v}\right)$ satisfies the following relations:

$$
d^{i} \alpha=0 \text { for } i<n, d^{n} \alpha=C^{n+1}(l) \zeta \text { and } d^{n+1} \alpha=C^{n+1}(k) \psi .
$$

As $C^{n+1}(l) \zeta$ and $C^{n+1}(k) \psi$ are the representatives of $l_{*} \partial(v) a$ and $k_{*} \partial\left(k\left(Y_{1}\right)\right) a$ respectively, it follows that $l_{*} \partial(v) a=k_{*} \partial\left(k\left(Y_{1}\right)\right) a$.

Thus the commutativity of 1$]$ is established.

ii) Commutativity of 2 immediately follows from $q_{2} j l=u$ in (4.7).

iii) Commutativity of 3 . Let $\tau: X \rightarrow C^{n+1}\left(Y_{2}\right)$ be a representative of $a \in$ $\pi_{n}\left(X, Y_{2}\right)$. Then by the definition $\partial\left(q_{2} j\right) a$ is represented by a morphism $\chi: X \rightarrow C^{n}\left(\Omega Y_{1}\right)$ determined by the following relations :

$$
C^{n+1}\left(q_{2} j\right)=\tau, d^{i} \xi=0 i<n \text { and } C^{n}(k) \psi=d^{n} \xi,
$$

where $\xi: X \rightarrow C^{n+1}\left(F_{v}\right)$ exists since $q_{2} j$ is a fibration.

On the other hand $\partial\left(k\left(Y_{1}\right)\right) v_{*} a$ is represented by a morphism $\psi: X \rightarrow C^{n}$ $\left(\Omega Y_{1}\right)$ determined by $C^{n}(i) \psi=d^{n} C^{n+1}\left(q_{1} j\right) \xi$, since $C^{n+1}\left(k\left(Y_{1}\right)\right) C^{n+1}\left(q_{1} j\right) \xi=C^{n+1}$ $(v) C^{n+1}\left(q_{2} j\right) \xi=C^{n+1}(v) \tau$ and $d^{i} C^{n+1}\left(q_{1} j\right) \xi=0$ for $i<n$.

It is easily verified that $C^{n}(i) \psi=C^{n}(i) \chi$. As $i$ is a monomorphism, we have $\psi=\chi$. This proves the commutativity of 3 .

Now we shall define an operation $\rho_{v_{*}}: \pi_{n}\left(X, F_{v}\right) \times \pi_{n}\left(X, \Omega Y_{1}\right) \rightarrow \pi_{n}\left(X, F_{v}\right)$ for any morphism $v: Y_{2} \rightarrow Y_{1}$.

Take $(a, b) \in \pi_{n}\left(X, F_{v}\right) \times \pi_{n}\left(X, \Omega Y_{1}\right)$ and let $\sigma: X \rightarrow C^{n+1}\left(F_{v}\right)$ and $\tau: X \rightarrow$ $C^{n+1}\left(\Omega Y_{1}\right)$ be the representatives of $a$ and $b$ respectively. Then from the Kan condition there exists a morphism $\rho: X \rightarrow C^{n+2}\left(F_{v}\right)$ such that $d^{n-1} \rho=\sigma, d^{n+1} \rho$ $=C^{n+1}(k) \tau$.

Then we define $\rho_{v_{*}}(a, b)$ by $\left\{d^{n} p\right\}$, that is to say, $\rho_{v_{*}}(a, b)=a \cdot k_{*}(b)$.

REMARK. Let $v: Y_{2} \rightarrow Y_{1}$ be a fibration. Consider the diagram 


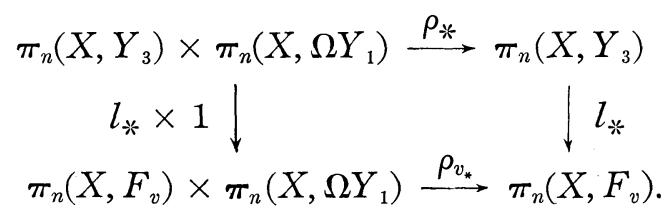

According to Theorem 4.8, $l_{*}$ is an isomorphism for $n \geqq 0$, and for $a \in$ $\pi_{n}\left(X, Y_{3}\right), b \in \pi_{n}(X, \Omega Y), l_{*} \rho_{*}(a, b)=l_{*}\left(a \cdot \partial(v) \partial^{1-}\left(k\left(Y_{1}\right)\right) b\right)=l_{*} a \cdot l_{*} \partial(v) \partial^{-1}\left(k\left(Y_{1}\right)\right)$ $b$; according to Theorem $4.9, l_{*} \partial(v) \partial^{-1}\left(k\left(Y_{1}\right)\right)=k_{*}$. Hence $l_{*} \rho_{*}(a, b)=l_{*} a \cdot k_{*}$ $(b)=\rho_{v_{*}}\left(l_{*} a \cdot b\right)=\rho_{v_{*}} \cdot\left(l_{*} \times 1\right)(a, b)$. Thus the above diagram is commutative. Therefore we may conclude that if $v: Y_{2} \rightarrow Y_{1}$ is a fibration, $\rho_{v_{*}}$ is equivalent to $\rho_{*}$ defined in $\S 2$.

THEOREM 4. 10.

(i) For $b_{1}, b_{2} \in \pi_{n}\left(X, \Omega Y_{1}\right), \rho_{v_{\star}}\left(k_{*} b_{1}, b_{2}\right)=k_{*}\left(b_{1} \cdot b_{2}\right)$.

(ii) If $a_{1}, a_{2} \in \pi_{n}\left(X, F_{v}\right)$, then $a_{1}=\rho_{v_{*}}\left(a_{2}, b\right)$ for some $b \in \pi_{n}\left(X, \Omega Y_{1}\right)$ if and only if $\left(q_{2} j\right)_{*} a_{1}=\left(q_{2} j\right)_{*} a_{2}$.

Proof. (i) and the necessity of (ii) are obvious. We only prove the sufficiency of (ii). Let $\sigma_{i}: X \rightarrow C^{n+1}\left(F_{v}\right)$ be a representative of $a_{i} \in \pi_{n}\left(X, F_{v}\right)$ $(i=1,2)$. From $\left(q_{2} j\right)_{*} a_{1}=\left(q_{2} j\right)_{*} a_{2}$, there exists a morphism $\xi: X \rightarrow C^{n+2}\left(Y_{2}\right)$ such that

$$
d^{i} \xi=0 \quad(i<n), d^{n} \xi=C^{n+1}\left(q_{2} j\right) \sigma_{1}, d^{n+1} \xi=C^{n+1}\left(q_{2} j\right) \sigma_{2} .
$$

Also since $q_{2} j: F_{v} \rightarrow Y_{2}$ is a fibration, there exists a morphism $\sigma: X \rightarrow C^{n+2}\left(F_{v}\right)$ such that

$$
d^{i} \sigma=0 \quad(i<n-1), d^{n} \sigma=\sigma_{1}, d^{n+} \sigma^{1}=\sigma_{2} \text { and } C^{n+2}\left(q_{2} j\right) \sigma=\xi .
$$

Then $d^{n+1} \sigma$ represents $a_{1}-a_{2} \in \pi_{n}\left(X, F_{v}\right)$ and $C^{n+1}\left(q_{2} j\right) d^{n-1} \sigma=d^{n-1} \xi=0$. Hence there exists a morphism $\tau: X \rightarrow C^{n+1}\left(\Omega Y_{1}\right)$ satisfying $C^{n+1}(k) \tau=d^{n-1} \sigma$. Since $C^{n+1}(k) d^{i} \tau=d^{i} d^{n-1} \quad \sigma=d^{n-2} d^{i} \sigma=0$ for $i<n-1$ and $k$ is a morphism, $d^{i} \tau$ $=0$ for $i<n-1$. Similary we may deduce that $d^{n-1} \tau=0$ and $d^{n} \tau=0$.

Now let $b$ be the homotopy class of $\tau$. Then the relations $d^{i} \sigma=0$ $(i<n-1), d^{n-1} \sigma=C^{n+1}(k) \tau, d^{n} \sigma=\sigma_{1}, d^{n+1} \sigma=\sigma_{2}$ imply $a_{1}=\rho_{v^{*}}\left(a_{2}, b\right)$.

Next we consider a monomorphism $u: Y_{3} \rightarrow Y_{2}$ in the fibration sequence

$$
Y_{3} \stackrel{u}{\longrightarrow} Y_{2} \stackrel{v}{\longrightarrow} Y_{1} \text {. }
$$

Denote the trace of $k\left(Y_{2}\right) \times u: C Y_{2} \times Y_{3} \rightarrow Y_{2} \times Y_{2}$ by $\left(F_{u}, h\right)$. Let $r_{1}: C Y_{2}$ $\times Y_{3} \rightarrow C Y_{2}$ and $r_{2}: C Y_{2} \times Y_{3} \rightarrow Y_{3}$ be projections, and let $s_{i}: Y_{2} \times Y_{2} \rightarrow Y_{2}$ $(i=1,2)$ be projection on the $i$-th factor. Then we have the following commutative diagram. 


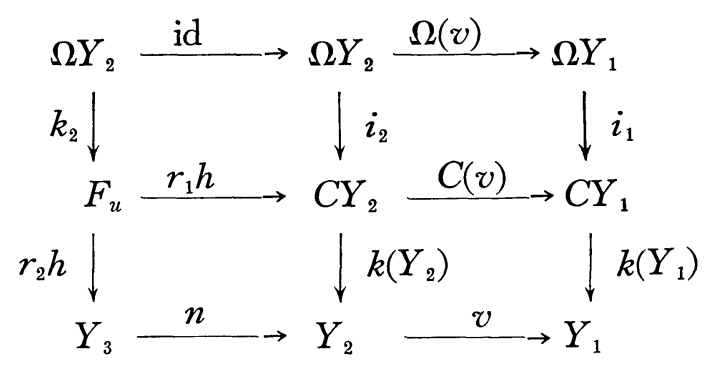

where the left column corresponds to the middle column in the diageam (4.7), and $\Omega(v): \Omega Y_{2} \rightarrow \Omega Y_{1}$ exists since $k\left(Y_{1}\right) C(v) i_{2}=v k\left(Y_{2}\right) i_{2}=0$ and $\left(\Omega\left(Y_{1}\right), i_{1}\right)$ is the kernel of $k\left(Y_{1}\right)$.

Now we consider a sequence $F_{u} \stackrel{h}{\longrightarrow} C Y_{2} \times Y_{3} \stackrel{C(v) \times u}{\longrightarrow} C Y_{1} \times Y_{2}$.

Since

$$
\begin{gathered}
k\left(Y_{1}\right) q_{1}(C(v) \times u) h=k\left(Y_{1}\right) C(v) r_{1} h=v k\left(Y_{2}\right) r_{1} h=v u r_{2} h=0 \\
v q_{2}(C(v) \times u) h=v u r_{2} h=0,
\end{gathered}
$$

and $F_{v}$ is the trace of $k\left(Y_{1}\right) \times v$, there exists a morphism $f: F_{u} \rightarrow F_{v}$ satisfying jf $=(C(v) \times u) h$. Then we have

$$
\begin{aligned}
& q_{1} j f k_{2}=q_{1}(C(v) \times u) h k_{2}=C(v) r_{1} h k_{2}=C(v) i_{2}, \\
& q_{2} j f k_{1}=q_{2}(C(v) \times u) h k_{2}=u r_{2} h k_{2}=0, \\
& q_{1} j k_{1} \Omega(v)=i_{1} \Omega(v)=C(v) i_{2}, \quad \text { where } k_{1}: \Omega Y_{1} \rightarrow F_{v}, \\
& q_{2} j k_{1} \Omega(v)=0 .
\end{aligned}
$$

Therefore by the property of the direct product, $j k_{1} \Omega(v)=j f k_{2}$ and hence $k_{1} \Omega(v)=f k_{2}$.

Thus we have the following commutative diagram;

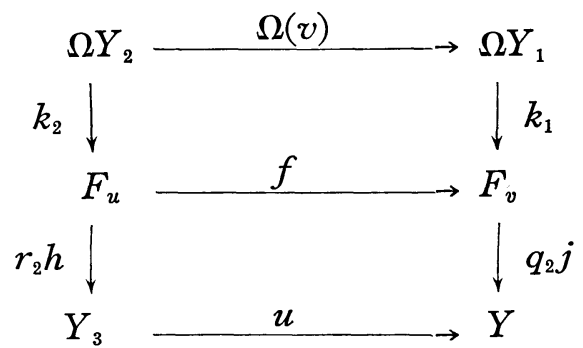

As $k\left(Y_{1}\right) C(v) r_{1} h=v u r_{1} h=0$ in (4.11), there also exists a morphism $t: F_{u}$ $\rightarrow \Omega Y_{1}$ satisfying $i_{1} t=C(v) r_{1} h$. Since $q_{1} j f=q_{1}(C(v) \times u) h=C(v) r_{1} h=i_{1} t, i_{1} t k_{2}$ $=q_{1} j f k_{2}=q_{1} j k_{2} \Omega(v)=i_{1} \Omega(v)$ and so $\Omega(v)=t k_{2}$.

Proposition 4.13. The following diagram is commutative. 


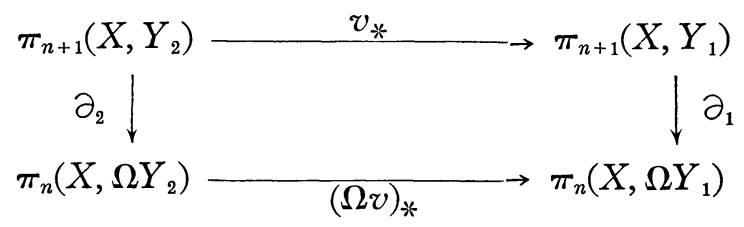

PROOF. Let $\tau: X \rightarrow C^{n+2}\left(Y_{2}\right)$ be a representative of $a \in \pi_{n+1}\left(X, Y_{2}\right)$. Then $\partial_{2} a$ is represented by a morphism $\varphi: X \rightarrow C^{n+1}\left(\Omega Y_{2}\right)$ determined by the relation $C^{n+2}\left(k\left(Y_{2}\right)\right) \sigma=\tau, d^{i} \sigma=0(0 \leqq i \leqq n), C^{n+1}\left(i_{2}\right) \varphi=d^{n+1} \sigma$ where $\sigma: X \rightarrow C^{n+2}\left(C Y_{2}\right)$ exists since $k\left(Y_{2}\right)$ is a fibration.

One has $C^{n+2}(\mathcal{V}) \tau=C^{n+2}(\mathcal{V}) C^{n+2}\left(k\left(Y_{2}\right)\right) \sigma=C^{n+2}\left(k\left(Y_{1}\right)\right) C^{n+2}(C(\mathcal{V})) \sigma$ and $d^{i} C^{n+2}$ $(v)_{\tau}=0$ for $i \neq n+1$. Hence $\partial_{1} v_{*} a$ is represented by $\psi: X \rightarrow C^{n+1}\left(\Omega Y_{1}\right)$ determined by $C^{n+1}\left(i_{1}\right) \psi=d^{n+1} C^{n+2}(C(v)) \sigma$. But $C^{n+1}\left(i_{1}\right) \psi=C^{n+1}(C(v)) d^{n+1} \sigma$ $=C^{n+1}(C(v)) C^{n+1}\left(i_{2}\right) \varphi=C^{n+1}\left(i_{1}\right) C^{n+1}(\Omega(v)) \varphi$. Therefore $\psi=C^{n+1}(\Omega(v)) \varphi$, for $C^{n+1}\left(i_{1}\right)$ is a monomorphism. Thus the commutativity in (5.13) is proved.

THEOREM 4.14. If $v: Y_{2} \rightarrow Y_{1}$ is a fibration, we have the following commutative diagram:

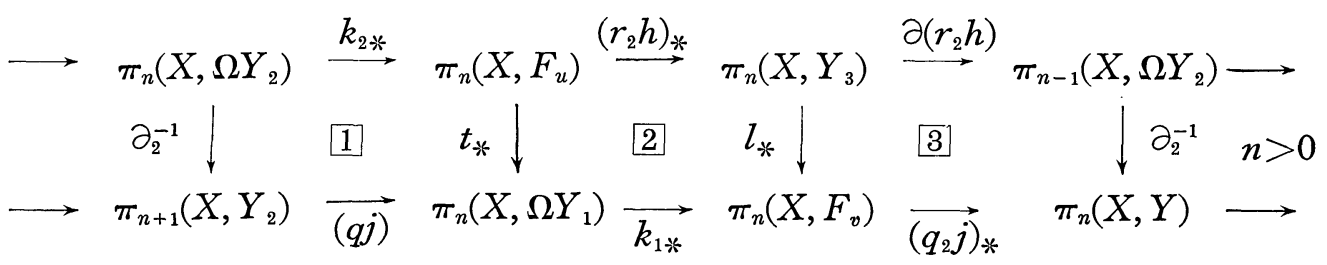

where the upper sequence is the Kan homotopy exact sequence for the fibration $r_{2} h: F_{u} \rightarrow Y_{3}$ and the lower is those for the fibration $q_{2} j: F_{v} \rightarrow Y_{2}$.

Proof. (i) Commutativity of 1 . From the commutativity of 3 in Theorem 4. 9, $\partial_{1} v_{*}=\partial\left(q_{2} j\right)$ and by Proposition (4.13) $\partial_{1} v_{*}=(\Omega v)_{*} \partial_{2}$ so that $\partial\left(q_{2} j\right)$ $=(\Omega v)_{*} \partial_{2}$. But $\Omega(v)=t k_{2}$. Hence $\partial\left(q_{2} j\right) \partial_{2}^{-1}=t_{*} k_{2 *}$.

(ii) Commutativity of 2 . It is sufficient to prove that $\left(r_{2} h\right)_{*}=\partial(v) \partial_{1}^{-1} t_{*}$.

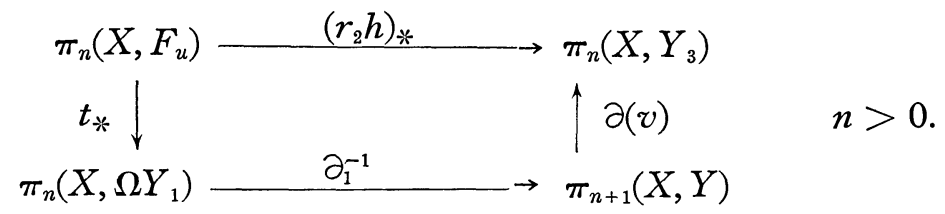

Let $a \in \pi_{n}\left(X, F_{u}\right)$ be represented by a morphism $\tau: X \rightarrow C^{n+1}\left(F_{u}\right)$ such that $d^{i} \tau$ $=0(0 \leqq i \leqq n)$. Since $\partial_{1}: \pi_{n+1}\left(X, Y_{1}\right) \rightarrow \pi_{n}\left(X, \Omega Y_{1}\right)$ is an isomorphism for $n>0$, there exists only one $c \in \pi_{n+1}\left(X, Y_{1}\right)$ such that $\partial_{1} c=t_{*} a$. Let $c \in \pi_{n+1}$ $\left(X, Y_{1}\right)$ be represented by a morphism $\sigma: X \rightarrow C^{n+1}\left(Y_{1}\right)$ such that $d^{i} \sigma=0(0 \leqq i$ $\leqq n+1$.) Then by the definition of $\partial_{1}$ there exists a morphism $\eta: X \rightarrow C^{n+2}$ 
$\left(C Y_{1}\right)$ such that $C^{n+2}\left(k\left(Y_{1}\right)\right) \eta=\sigma, d^{i} \eta=0(0 \leqq i \leqq n), d^{n+1} \eta=C^{n+1}\left(i_{1} t\right) \tau$.

Therefore $\partial(v) \partial_{1}^{-1} t_{*} a$ is represented by a morphism $\xi: X \rightarrow C^{n+1}\left(Y_{3}\right)$ determined by the relation $C^{n+2}(v) \chi=\sigma, d^{i} \chi=0(0 \leqq i \leqq n), d^{n+1} \chi=C^{n+1}(u) \xi$, where $\chi: X \rightarrow C^{n+2}\left(Y_{2}\right)$ exists since $v: Y_{2} \rightarrow Y_{1}$ is a fibration. On the other hand $\left(r_{2} h\right)_{*} a$ is represented by $C^{n+1}\left(r_{2} h\right)_{\tau}: X \rightarrow Y_{3}$.

Now consider $C^{n+1}\left(r_{1} h\right)_{\tau}: X \rightarrow C^{n+1}\left(C Y_{2}\right)$. It follows from $i_{1} t=C(v) r_{1} h$ that $C^{n+1}(C(\mathcal{v})) C^{n+1}\left(r_{1} h\right)_{\tau}=C^{n+1}\left(i_{1} t\right) \tau=d^{n+1} \eta$. If we regard $C^{n+1}\left(r_{1} h\right) \tau$ as a $(n+1)$ -simplex of $K_{*}\left(X, Y_{2}\right)$, i.e. $C^{n+1}\left(r_{1} h\right)_{\tau}: X \rightarrow C^{n+2}\left(Y_{2}\right)$, we have $C^{n+2}(v) C^{n+1}\left(r_{1} h\right) \tau$ $=d^{n+1} \eta$ where $\eta$ is regarded as a $(n+2)$-simplex of $K_{*}\left(X, Y_{1}\right)$.

On the other hand $\sigma=C^{n+2}\left(k\left(Y_{1}\right)\right) \eta=d^{n+2} \eta$ and $d^{i} \eta=0(0 \leqq i \leqq n)$. Hence $C^{n+2}(v) C^{n+1}\left(r_{1} h\right) \tau \sim \sigma\left(C^{n+2}(v) C^{n+1}\left(r_{1} h\right) \tau\right.$ is semi-simplicial homotopic to $\left.\sigma\right)$. But $d^{n+1} C^{n+1}\left(r_{1} h\right) \tau=C^{n+1}\left(k\left(Y_{2}\right)\right) C^{n+1}\left(r_{1} h\right) \tau=C^{n+1}(u) C^{n+1}\left(r_{2} h\right) \tau$ and $d^{i} C^{n+1}\left(r_{1} h\right) \tau=0$ $(0 \leqq i \leqq n)$. Hence by the definition of $\partial(v): \pi_{n+1}\left(X, Y_{1}\right) \rightarrow \pi_{n}\left(X, Y_{3}\right)$, we may regard $C^{n+1}\left(r_{2} h\right) \tau$ as a representative of $\partial(v) c$. Thus $\left(r_{2} h\right)_{*}=\partial(v) \partial_{1}^{-1} t_{*}$.

(iii) Commutativity of 3 . Let $a \in \pi_{n}\left(X, Y_{3}\right)$ be represented by a morphism $\tau: X \rightarrow C^{n+1}\left(Y_{3}\right)$ such that $d^{i} \tau=0(0 \leqq i \leqq n)$. By Theorem 4.3, $r_{2} h: F_{u} \rightarrow Y_{3}$ is also a fibration and $\partial\left(r_{2} h\right) a$ is represented by a morphism $\chi: X \rightarrow C^{n}\left(\Omega Y_{2}\right)$ determined by the relation $C^{n+1}\left(r_{2} h\right) \sigma=\tau, d^{i} \sigma=0 \quad(0 \leqq i \leqq n), \quad C^{n}\left(k_{2}\right) \chi=d^{n} \sigma$, where $\sigma: X \rightarrow C^{n+1}\left(F_{u}\right)$ exists since $r_{2} h$ is a fibration.

Since $C^{n+1}(u) \tau=C^{n+1}(u) C^{n+1}\left(r_{2} h\right) \sigma=C^{n+1}\left(k\left(Y_{2}\right)\right) C^{n+1}\left(r_{1} h\right) \sigma$ and $d^{i} C^{n+1}\left(r_{1} h\right) \sigma$ $=0(0 \leqq i \leqq n)$, it follows from the definition of $\partial_{2}: \pi_{n}\left(X, Y_{2}\right) \rightarrow \pi_{n-1}\left(X, \Omega Y_{2}\right)$ that a morphism $\psi: X \rightarrow C^{n}\left(\Omega Y_{1}\right)$ determined by $d^{n} C^{n+1}\left(r_{1} h\right) \sigma=C^{n}\left(i_{2}\right) \psi$ represents an element of $\pi_{n-1}\left(X, \Omega Y_{2}\right)$. Then $C^{n}\left(i_{2}\right) \psi=d^{n} C^{n+1}\left(r_{1} h\right) \sigma=C^{n}\left(r_{1} h\right) d^{n} \sigma=$ $C^{n}\left(r_{1} h\right) C^{n}\left(k_{2}\right) \chi=C^{n}\left(i_{2}\right) \chi$, where $\left(r_{1} h\right) k_{2}=i_{2}$ follows from (4. 11). As $C^{n}\left(i_{2}\right)$ is monomorphic this implies $\psi=\chi$ and $\partial_{2} u_{*}=\partial\left(r_{2} h\right)$ follows.

On the other hand $q_{2} j l=u$ and hence $\partial_{2}\left(q_{2} j\right)_{*} l_{*}=\partial\left(r_{2} h\right)$.

From Theorems 4.8 and 4.14 and the five lemma we obtain the following theorem:

THEOREM 4. 15. If $Y_{3} \stackrel{u}{\longrightarrow} Y_{2} \stackrel{v}{\longrightarrow} Y_{1}$ is a fibration sequence, then

$$
t_{*}: \pi_{n}\left(X, F_{u}\right) \rightarrow \pi_{n}\left(X, \Omega Y_{1}\right)
$$

is an isomorphism for $n>1$.

Here we note that Theorem 4.15 corresponds to Proposition 4.7 in [2].

5. The dual statements. Finally we shall consider the dual statement. A dual standard construction in $\Omega$ is a triple $\{C, k, p\}$ consisting of a covariant functor $C: \mathscr{\kappa} \rightarrow \Re$, and of functor morphisms $k: I \rightarrow C$ and $p: C C \rightarrow C$, such that the axioms

$$
\begin{aligned}
& p \circ(k * C)=p *(C * k)=\text { identity, } \\
& p \circ(p * C)=p \circ(C * p)
\end{aligned}
$$

are satisfied. 
The functor $F^{*}=\left(F^{n}, d^{i}, S^{i}\right)$ belonging to a dual standard construction has a dual semi-simplicial structure, i. e. the faces and degeneracy morphisms

$$
\begin{gathered}
d_{n}^{i}: F^{n-1} \longrightarrow F^{n} \\
s_{n}^{i}: F^{n+1} \longrightarrow F^{n}
\end{gathered}
$$

go into the opposite direction and satisfy the relation dual to (a)-(e) in $\S 2$. Let $K_{*}(X, Y)$ be the semi-simplicial complex induced by a dual standard construction $\{C, k, p\}$, i. e.

$$
K_{*}(X, Y)=\operatorname{Hom}\left(F^{*}(X), Y\right)=\left(\operatorname{Hom}\left(F^{n}(X), Y\right), d^{i}, s^{i}\right) .
$$

Then we have the Kan homotopy groups $\pi_{n}(X, Y)=\pi_{n}\left(K_{*}(X, Y)\right.$. Here we shall assume that all the $K_{*}(X, Y)$ satisfy the Kan condition.

DEFINITION 2. $1^{\prime}([5])$. A morphism $u: X_{1} \rightarrow X_{2}$ is called a cofibration if the induced semi-simplicial map

$$
u^{*}: K_{*}\left(X_{2}, Y\right) \rightarrow K_{*}\left(X_{1}, Y\right)
$$

is a semi-simplicial fibration (cf. [6]).

If $C$ commutes with cokernels (i. e. $C($ Coker $u)=$ Coker $C(u)$, then the cokernel $X_{3}$ of $u$ will be called cofibre of $u$.

Propsition. 2. 2' (Huber [5]).

1) The morphism $k(X): X \rightarrow C X$ is a cofibration.

$$
\pi_{n}(C X, Y)=0 \quad \text { for } n \geqq 0 .
$$

If we denote $\Sigma X$ the cokernel of $k(X)$ and $C$ commutes $\Sigma X$, then

Proposition. 2. 3' (Huber [5])

$$
\partial(k(X)): \pi_{n+1}(X, Y) \rightarrow \pi_{n}(\Sigma X, Y) \text { is an isomorphism for } n>0 .
$$

In the following we shall assume the existences of cofibres.

Let $u: X_{1} \rightarrow X_{2}$ be a cofibration in $\mathcal{A}$ and let $\left(X_{3}, v\right)$ its cofibre. By Proposition $2.2^{\prime}, k\left(X_{1}\right): X_{1} \rightarrow C X_{1}$ is a cofibration. Then as dual in $\S 3$ we may define a co-operation

$$
\rho^{*}: \pi_{n}\left(X_{3}, Y\right) \times \pi_{n}\left(\Sigma X_{1}, Y\right) \rightarrow \pi_{n}\left(X_{3}, Y\right) .
$$

\section{THEOREM $3.1^{\prime}$.}

2)

$$
\begin{aligned}
& \rho^{*}(a, 0)=a \text {, where } a \in \pi_{n}\left(X_{3}, Y\right) \text { and } 0 \in \pi_{n}\left(\Sigma X_{1}, Y\right) \text { denotes the unit } \\
& \text { element. } \\
& \rho^{*}\left(a, b_{1} \cdot b_{2}\right)=\rho^{*}\left(\rho^{*}\left(a, b_{1}\right), b_{2}\right) \text {, where } a \in \pi_{n}\left(X_{3}, Y\right), b_{i} \in \pi_{n}\left(\Sigma X_{i}, Y\right) \\
& (i=1,2) \text {. }
\end{aligned}
$$

Let $\Omega$ be the category of topological spaces with base points, $\operatorname{Hom}(X, Y)$ being the set of base points preserving continuous maps $X \rightarrow Y$, with the natural 
rule of composition. Then a dual standard construction $\{C, k, p\}$ in $\Re$ was defined in [5] as the cone construction:

$$
C X=X \times I / X \times\{0\} \cup\{*\} \times I,
$$

$I$ denoting the real interval $0 \leqq t \leqq 1$, with the base point 0 .

Again let $\mathscr{R}$ be a general category and suppose that $\Re$ has inverse products. Here we recall the definition of an inverse product in [3].

An inverse product of the object $A_{1}, A_{2}$ is an object $A_{1} * A_{2}$ and a system of morphisms $q_{j}: A_{i} \rightarrow A_{1} * A_{2}(j=1,2)$ with the property

(I) For any object $X$ of $f_{t}$ and any system of morphisms $f_{j}: A_{j} \rightarrow A_{1} * A_{2}$ $(j=1,2)$, there exists a unique morphism $f: A_{1} * A_{2} \rightarrow X$ with $f q_{j}=f_{j}(j=1,2$.)

The morphisms $q_{j}$ are called the injections into $A_{1} * A_{2}$; and the morphisms $f_{j}$ are called the component of $f$; we write $f=\left\langle f_{1}, f_{2}\right\rangle$, so that

$$
<f_{1}, f_{2}>q_{j}=f_{j}
$$

Let $f_{i}: B \rightarrow A_{i}, i=1,2$, be morphisms, then the morphism $<q_{1} f_{1}, q_{2} f_{2}>$ : $B * B \rightarrow A_{1} * A_{2}$ will be written $f_{1} * f_{2}$. Then we have $\left(f_{1} * f_{2}\right) q_{i}^{\prime}=q_{i} f_{i}(i=1,2)$, where $q_{j}^{\prime}: B \rightarrow B * B, j=1,2$, are injections.

DEFinition 4. $1^{\prime}$. The trace of $f_{1} * f_{2}: B * B \rightarrow A_{1} * A_{2}$ is a pair $(Q, \rho)$ consisting of an object and an epimorphism satisfy the conditions

$$
\rho\left(f_{1} * f_{2}\right) q_{1}^{\prime}=\rho\left(f_{1} * f_{2}\right) q_{2}^{\prime} \text { (or equivalently } \rho q_{1} f_{1}=\rho q_{2} f_{2} \text { ) }
$$

(ii) if $D$ is any object of $\Omega$ and $h: A_{1} * A_{2} \rightarrow D$ is a morphism with the property $h\left(f_{1} * f_{2}\right) q_{1}^{\prime}=h\left(f_{1} * f_{2}\right) q_{2}^{\prime}$ (or equivalently $h q_{1} f_{1}=h q_{2} f_{2}$ ), then $h$ admits a unique factorization $A_{1} * A_{2} \stackrel{\rho}{\rightarrow} Q \rightarrow D$.

We assume that the functor $C$ in consideration is $I$-functor in the sense of [3], i. e. $C\left(A_{1} * A_{2}\right)=C\left(A_{1}\right) * C\left(A_{2}\right)$ and $C\left(q_{i}\right): C\left(A_{i}\right) \rightarrow C\left(A_{1} * A_{2}\right), i=1,2$, are injections. Moreover we assume that if $g: B \rightarrow A$ is an epimorphism, then $C(g): C(B) \rightarrow C(A)$ is so.

Let $k\left(X_{1}\right): X_{1} \rightarrow C X_{1}$ be a morphism in Proposition 2.2' and $v: X_{1} \rightarrow X_{2}$ any morphism. Then we denote the trace of $k\left(X_{1}\right) * v$ by $\left(F^{v}, p\right)$. Let $q_{1}: C X_{1}$ $\rightarrow C X_{1} * X_{2}$ and $q_{2}: X_{2} \rightarrow C X_{1} * X_{2}$ be injections, then we have the following commutative diagram;

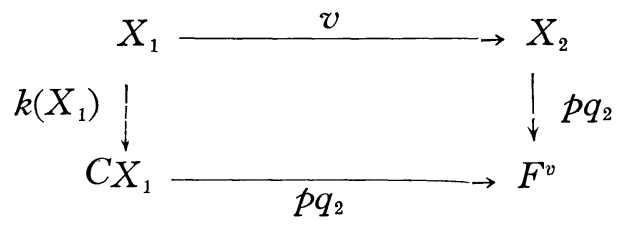

The proofs of the following theorems are quite dual to that of the preceeding ones and we shall omit. 
THEOREM 4. $3^{\prime} . p q_{2}: X_{2} \rightarrow F^{v}$ is a cofibration.

THEOREM 4. 4'. If $v: X_{1} \rightarrow X_{2}$ is a cofibration, $p q_{1}: C X_{1} \rightarrow F^{v}$ is so.

THEOREM 4. $5^{\prime}$. The cofibre of cofibration $p q_{2}: X_{2} \rightarrow F^{v}$ is equivalent with $\Sigma X_{1}$, the cofibre of $k(X): X_{1} \rightarrow C X_{1}$.

THEOREM 4. $6^{\prime}$. If a sequence $X_{1} \rightarrow X_{2} \rightarrow X_{3}$ is a cofibration sequence, then a sequence $C X_{1} \rightarrow F^{v} \rightarrow X_{3}$ is so.

If $v: X_{1} \rightarrow X_{2}$ is a cofibration with cofibre $X_{3}$, then the following diagram is commutative.

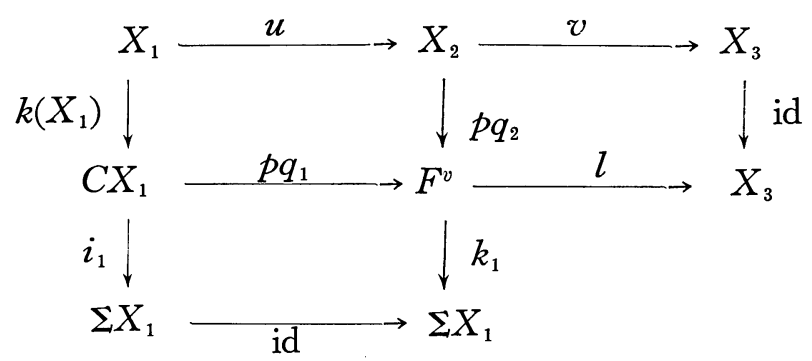

THEOREM 4.8'. If a morphism $v: X_{1} \rightarrow X_{2}$ is a cofibration with cofibre $X_{3}$, then $l_{*}: \pi_{n}\left(X_{3}, Y\right) \rightarrow \pi_{n}\left(F^{v}, Y\right)$ is an isomorphism for $n \geqq 0$.

THEOREM 4.9'. If $v: X_{1} \rightarrow X_{2}$ is a cofibration with cofibre $X_{3}$, then the following diagram is commutative :

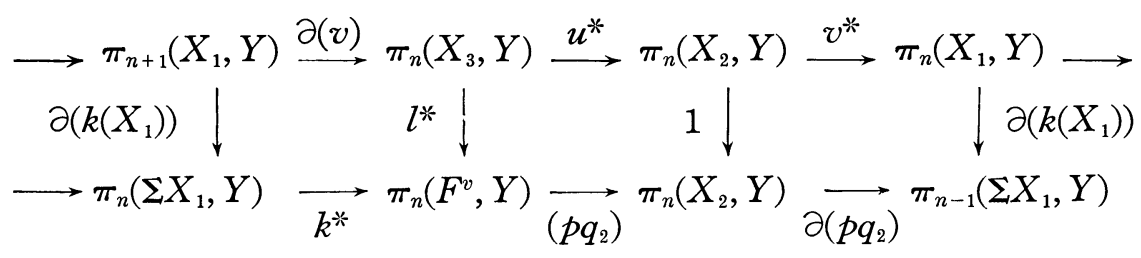

where the upper sequence is the Kan homotopy exact sequence of a cofibration $v: X_{1} \rightarrow X_{2}$ and the lower is that of a cofibration $p q_{2}: X_{2} \rightarrow F^{v}$.

For any morphism $v: X_{1} \rightarrow X_{2}$ co-operation $\rho_{v}^{*}: \pi_{n}\left(F^{v}, Y\right) \times \pi_{n}\left(\Sigma X_{1}, Y\right) \rightarrow$ $\pi_{n}\left(F^{v}, Y\right)$ is defined by

$$
\rho_{v}^{*}(a, b)=a \cdot k^{*}(b), \quad a \in \pi_{n}\left(F^{v}, Y\right), b \in \pi_{n}\left(\Sigma X_{1}, Y\right) .
$$

If $v: X_{1} \rightarrow X_{2}$ is a cofibration, we may show that $\rho_{v}^{*}$ is equivalent with $\rho^{*}$.

THEOREM 4. $10^{\prime}$.

(i) For $b_{1}, b_{2} \in \pi_{n}\left(\Sigma X_{1}, Y\right), \rho_{v}^{*}\left(k^{*}\left(b_{1}\right), b_{2}\right)=k^{*}\left(b_{1} \cdot b_{2}\right)$

(ii) If $a_{1}, a_{2} \in \pi_{n}\left(F^{v}, Y\right)$, then $a=\rho_{v}^{*}\left(a_{2}, b\right)$ for some $b \in \pi_{n}\left(\Sigma X_{1}, Y\right)$ if and 
any if $\left(p q_{2}\right) * a_{1}=\left(p q_{2}\right) * a_{2}$.

Let $X_{1} \stackrel{v}{\longrightarrow} X_{2} \stackrel{u}{\longrightarrow} X_{3}$ be a cofibration sequence. We denote the trace of $k\left(X_{2}\right) * u: X_{2} * X_{2} \longrightarrow C X_{2} * X_{3}$ by $\left(F^{u}, h\right)$. Let $r_{1}, r_{2}: C X_{2}, X_{3} \longrightarrow C X_{2} * X_{3}$ be injections. Then (4.11) and (4.12) may be dualized as follows.

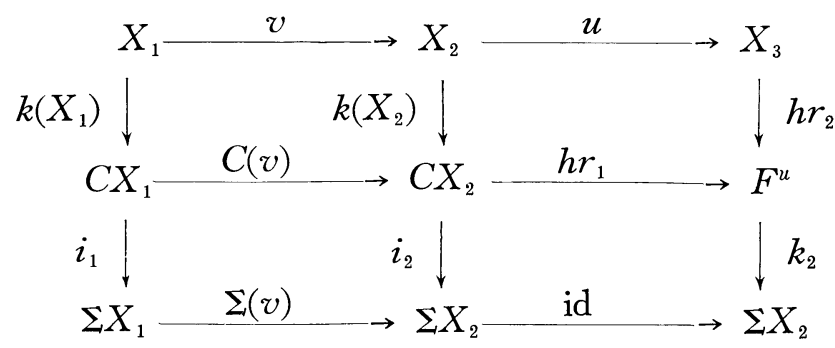

$\left(4.12^{\prime}\right)$

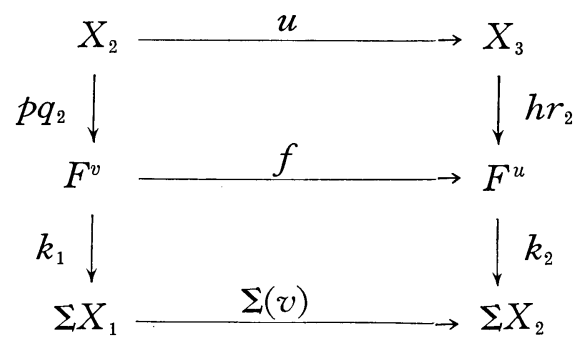

Then there exists a morphism $t: \Sigma X_{1} \rightarrow F^{u}$ such that $t i_{1}=h r_{1} \cdot C(v)$. Also $\Sigma(v)=k_{2} t$.

Proposition 4.13'. The following diagram is commutative.

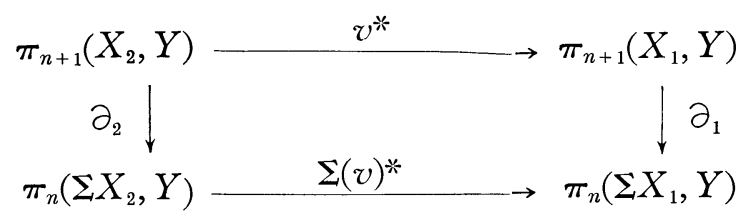

THEOREM 4.14.' If $v: X_{1} \rightarrow X_{2}$ is a cofibration with cofibre $X_{3}$, then we have the following commutative diagram.

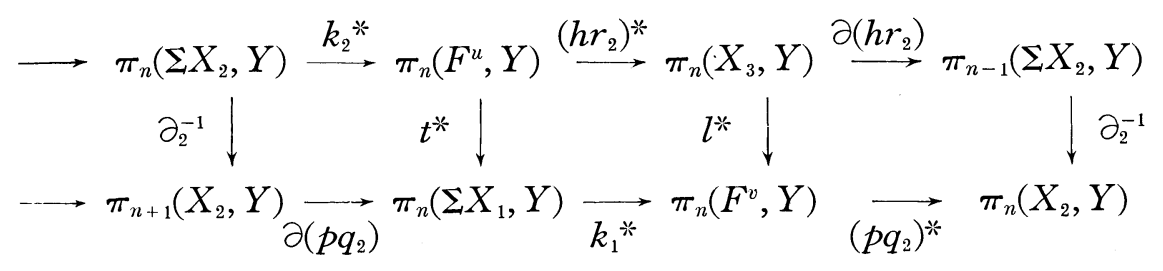


where the upper sequence is the Kan homotopy exact sequence of a cofibration $h r_{2}: X_{3} \rightarrow F^{u}$ and the lower is that of a cofibration $p q_{2}: X_{2} \rightarrow F^{v}$.

THEOREM 4. 15'. If $X_{1} \stackrel{v}{\longrightarrow} X_{2} \stackrel{u}{\longrightarrow} X_{3}$ is a cofibration sequence then

$$
t^{*}: \pi_{n}\left(F^{u}, Y\right) \rightarrow \pi_{n}\left(\Sigma X_{1}, Y\right)
$$

is an isomorphism for $n>1$.

\section{REFERENCES}

[1] B. EckmanN and P. J. Hilton, Homotopie et dualité, C. R. Acad. Sci., Paris, 246 (1958), 2444-2447, 2555-2558, 2991-2993.

[2] 1 , Operators and cooperators in homotopy theory, Math. Ann., 141(1960),

[3] $\frac{-255}{-}$, Group-like structures in general categories, Math. Ann., 145(1962), 227

[4] R. Godement, Théorie des faisceaux, Paris, Hermann, 1958.

[ 5 ] P. J. Huber, Homotopy theory in general categories, Math. Ann., 144(1961), 361-385.

[6] D. M. KAN, A combinatorial definition of homotopy groups, Ann. of Math., 67(1958), $282-312$.

HIROSAKI UNIVERSITY. 